\title{
Hodge numbers of a double octic with non-isolated singularities
}

\author{
by SŁawomir Cynk (Kraków)
}

\begin{abstract}
If $B$ is a surface in $\mathbb{P}^{3}$ of degree 8 which is the union of two smooth surfaces intersecting transversally then the double covering of $\mathbb{P}^{3}$ branched along $B$ has a non-singular model which is a Calabi-Yau manifold. The aim of this note is to compute the Hodge numbers of this manifold.
\end{abstract}

1. Introduction. Let $B$ be a surface of degree 8 in $\mathbb{P}^{3}$. Assume that $B$ is the union of two smooth surfaces $B_{1}$ and $B_{2}$ of degrees $d$ and $e$ respectively intersecting transversally along a smooth curve $C$. Denote by $\sigma: \widetilde{\mathbb{P}}^{3} \rightarrow \mathbb{P}^{3}$ the blow-up of $\mathbb{P}^{3}$ with center $C$ and consider the double covering $\pi: X \rightarrow \widetilde{\mathbb{P}}^{3}$ of $\widetilde{\mathbb{P}}^{3}$ branched along the strict transform $\widetilde{B}$ of $B$.

From [5] it follows that in this situation $X$ is a Calabi-Yau manifold and $e(X)=8-\left(d^{3}-4 d^{2}+6 d\right)-\left(e^{3}-4 e^{2}+6 e\right)-8 d e$. However it is of great interest to calculate not only the Euler characteristic but also the cohomology groups or equivalently the Hodge numbers of $X$. For a Calabi-Yau variety only two Hodge numbers are interesting: $h^{1,1}$ and $h^{1,2}$ - the others are obvious. We have moreover the following formula:

$$
e(X)=2\left(h^{1,1}-h^{1,2}\right) .
$$

These Hodge numbers have deep topological characterizations:

- $h^{1,1}$ is equal to the rank of the Picard group Pic $X$,

- $h^{1,2}$ is equal to the number of deformations of $X$.

In general it is very difficult to calculate the Hodge numbers of a double solid. Some methods are known only in very special cases (see $[2,6]$ ). In $[3]$ we gave an elementary proof of the Clemens formula for the Hodge numbers of a nodal double solid. We shall apply the method introduced there. This shows

2000 Mathematics Subject Classification: Primary 14J17; Secondary 14J32.

Key words and phrases: Hodge numbers, Calabi-Yau manifolds, double solids, surface singularities.

Partially supported by KBN grant no 2P03A 08310. 
that it may be of use also in the case of a double octic with non-isolated singularities in the branch locus.

2. Conormal bundle of $\pi^{*} \widetilde{B}$ in $X$. Denote by $E$ the exceptional divisor of $\sigma$ and by $\widetilde{B}_{i}$ the strict transform of $B_{i}$. Clearly $\sigma \mid \widetilde{B}_{i} \rightarrow B_{i}$ is an isomorphism. Since $\widetilde{B}$ is an even element of $\operatorname{Pic}\left(\widetilde{\mathbb{P}}^{3}\right)$ we can define the line bundle $\mathcal{L}=\mathcal{O}_{\widetilde{\mathbb{P}} 3}\left(\frac{1}{2} \widetilde{B}\right)$. The aim of this section is to study the line bundle $\pi^{*}\left(\mathcal{O}_{\widetilde{B}} \otimes \mathcal{L}^{-1}\right)$ which is dual to the normal bundle of $\pi^{*} \widetilde{B}$ in $X$.

From the definition of $\mathcal{L}$ we have $\mathcal{L}^{-1}=\sigma^{*} \mathcal{O}_{\mathbb{P}^{3}}(-4) \otimes \mathcal{O}_{\widetilde{\mathbb{P}} 3}(E)$ and so $H^{i}\left(\mathcal{L}^{-1} \otimes \mathcal{O}_{\widetilde{B}}\right) \cong H^{i}\left(\mathcal{O}_{\mathbb{P}^{3}}(e-4) \otimes \mathcal{O}_{B_{1}}\right) \oplus H^{i}\left(\mathcal{O}_{\mathbb{P}^{3}}(d-4) \otimes \mathcal{O}_{B_{2}}\right)$. Using the last formula we easily get

LEMMA 2.1.

$$
\begin{aligned}
& H^{0}\left(\mathcal{L}^{-1} \otimes \mathcal{O}_{\widetilde{B}}\right) \cong \begin{cases}\mathbb{C}^{10} & \text { if } d=1, e=7, \\
\mathbb{C}^{9} & \text { if } d=2, e=6, \\
\mathbb{C}^{4} & \text { if } d=3, e=5, \\
\mathbb{C}^{2} & \text { if } d=4, e=4,\end{cases} \\
& H^{1}\left(\mathcal{L}^{-1} \otimes \mathcal{O}_{\widetilde{B}}\right)=0, \\
& H^{2}\left(\mathcal{L}^{-1} \otimes \mathcal{O}_{\widetilde{B}}\right) \cong \begin{cases}\mathbb{C}^{84} & \text { if } d=1, e=7, \\
\mathbb{C}^{35} & \text { if } d=2, e=6, \\
\mathbb{C}^{10} & \text { if } d=3, e=5, \\
\mathbb{C}^{2} & \text { if } d=4, e=4 .\end{cases}
\end{aligned}
$$

\section{Cohomology of $\pi^{*} \Omega_{\widetilde{\mathbb{p}} 3}^{1}$}

LEMMA 3.1.

$$
H^{i}\left(\Omega_{\widetilde{\mathbb{P}}^{3}}^{1}\right) \cong \begin{cases}0 & \text { if } i=0,3, \\ \mathbb{C}^{2} & \text { if } i=1, \\ \mathbb{C}^{g} & \text { if } i=2,\end{cases}
$$

where the genus $g$ of $C$ is $2 d e+1$.

Pro of. Consider the following long exact sequence:

$$
0 \rightarrow \sigma^{*} \Omega_{\mathbb{P}^{3}}^{1} \rightarrow \Omega_{\widetilde{\mathbb{P}}^{3}}^{1} \rightarrow \Omega_{\mathbb{\mathbb { P }}^{3} / \mathbb{P}^{3}}^{1} \rightarrow 0 .
$$

Following [7, Thm. II.8.24] we can identify $E$ with the projectivization $\mathbb{P}\left(\mathcal{N}_{C \mid \mathbb{P}^{3}}^{\vee}\right)$ of the conormal bundle $\mathcal{N}_{C \mid \mathbb{P}^{3}}^{\vee}$ of $C$ in $\mathbb{P}^{3}$. Since in this situation $\Omega_{\widetilde{\mathbb{P}}^{3} / \mathbb{P}^{3}}^{1} \cong \Omega_{E / C}^{1}$ and (by [7, Ex. III.8.4]) $\Omega_{E / C}^{1} \cong \sigma^{*}\left(\bigwedge^{2} \mathcal{N}_{C \mid \mathbb{P}^{3}}^{\vee}\right) \otimes \mathcal{O}_{E}(-2)$, using the projection formula and again [7, Ex. III.8.4] we get

$$
\sigma_{*} \Omega_{\mathbb{\mathbb { P }}^{3} / \mathbb{P}^{3}}^{1} \cong \sigma_{*} \Omega_{E / C}^{1} \cong\left(\bigwedge^{2} \mathcal{N}_{C \mid \mathbb{P}^{3}}^{\vee}\right) \otimes \sigma_{*} \mathcal{O}_{E}(-2)=0
$$


and

$$
\begin{aligned}
R^{1} \sigma_{*} \Omega_{\mathbb{P}^{3} / \mathbb{P}^{3}}^{1} & \cong\left(\bigwedge^{2} \mathcal{N}_{C \mid \mathbb{P}^{3}}^{\vee}\right) \otimes R^{1} \sigma_{*} \mathcal{O}_{E}(-2) \\
& \cong\left(\bigwedge^{2} \mathcal{N}_{C \mid \mathbb{P}^{3}}^{\vee}\right) \otimes\left(\sigma_{*} \mathcal{O}_{E}\right)^{\vee} \otimes\left(\bigwedge^{2} \mathcal{N}_{C \mid \mathbb{P}^{3}}^{\vee}\right)^{\vee} \\
& \cong\left(\sigma_{*} \mathcal{O}_{E}\right)^{\vee} \cong \mathcal{O}_{C} .
\end{aligned}
$$

The direct image functor applied to the short exact sequence (1) yields

$$
\sigma_{*} \Omega_{\mathbb{P}^{3}}^{1} \cong \Omega_{\mathbb{P}^{3}}^{1} \text { and } \quad R^{1} \sigma_{*} \Omega_{\mathbb{P}^{3}}^{1} \cong \mathcal{O}_{C} .
$$

The Leray spectral sequence $H^{p}\left(R^{q}\left(\sigma_{*} \Omega_{\mathbb{\mathbb { P }}^{3}}^{1}\right)\right)$ has the following terms:

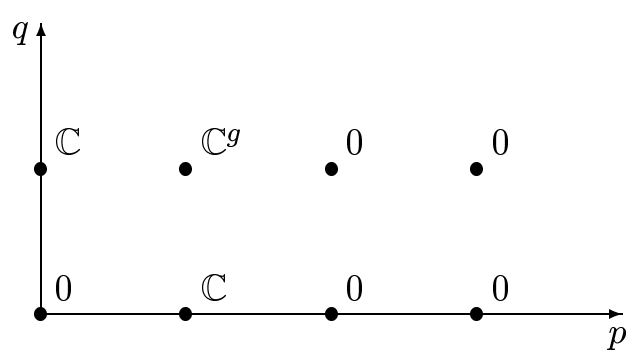

where $g=2 d e+1$ is the genus of $C$. The above sequence degenerates and the lemma follows.

LEMMA 3.2.

$$
\begin{aligned}
& H^{0}\left(\Omega_{\mathbb{\mathbb { P }}^{3}}^{1} \otimes \mathcal{L}^{-1}\right)=0, \\
& H^{1}\left(\Omega_{\widetilde{\mathbb{P}}^{3}}^{1} \otimes \mathcal{L}^{-1}\right) \cong \begin{cases}\mathbb{C}^{10} & \text { if } d=1, e=7, \\
\mathbb{C}^{9} & \text { if } d=2, e=6, \\
\mathbb{C}^{4} & \text { if } d=3, e=5, \\
\mathbb{C}^{2} & \text { if } d=4, e=4 .\end{cases}
\end{aligned}
$$

Proof. Tensoring the exact sequence (1) with $\mathcal{L}^{-1}$ we get

$$
0 \rightarrow \sigma^{*} \Omega_{\mathbb{P}^{3}}^{1} \otimes \mathcal{L}^{-1} \rightarrow \Omega_{\widetilde{\mathbb{P}}^{3}}^{1} \otimes \mathcal{L}^{-1} \rightarrow \Omega_{\widetilde{\mathbb{P}}^{3} / \mathbb{P}^{3}}^{1} \otimes \mathcal{L}^{-1} \rightarrow 0 .
$$

In this situation

$$
\left(\sigma^{*} \Omega_{\mathbb{P}^{3}}^{1}\right) \otimes \mathcal{L}^{-1} \cong\left(\sigma^{*} \Omega_{\mathbb{P}^{3}}^{1}(-4)\right) \otimes \mathcal{O}_{\widetilde{\mathbb{P}}^{3}}(E)
$$

and

$$
\begin{aligned}
\Omega_{\mathbb{\mathbb { P }}^{3} / \mathbb{P}^{3}}^{1} \otimes \mathcal{L}^{-1} & \cong \Omega_{E / C}^{1} \otimes \sigma^{*} \mathcal{O}_{\mathbb{P}^{3}}(-4) \otimes \mathcal{O}_{\widetilde{\mathbb{P}}^{3}}(E) \\
& \cong \sigma^{*}\left(\bigwedge^{2} \mathcal{N}_{C \mid \mathbb{P}^{3}}^{\vee}\right) \otimes \mathcal{O}_{E}(-2) \otimes \sigma^{*} \mathcal{O}_{\mathbb{P}^{3}}(-4) \otimes \mathcal{O}_{\widetilde{\mathbb{P}}^{3}}(E) \otimes \mathcal{O}_{E} \\
& \cong \sigma^{*}\left(\bigwedge^{2} \mathcal{N}_{C \mid \mathbb{P}^{3}}^{\vee} \otimes \mathcal{O}_{\mathbb{P}^{3}}(-4)\right) \otimes \mathcal{O}_{E}(-3)
\end{aligned}
$$

because $\mathcal{O}_{\widetilde{\mathbb{P}}^{3}}(E) \otimes \mathcal{O}_{E} \cong \mathcal{N}_{E \mid \widetilde{\mathbb{P}}^{3}} \cong \mathcal{O}_{E}(-1)$ by [7, Thm. II.8.24]. 
By the projection formula,

$$
\begin{aligned}
\sigma_{*}\left(\left(\sigma^{*} \Omega_{\mathbb{P}^{3}}^{1}\right) \otimes \mathcal{L}^{-1}\right) & \cong \Omega_{\mathbb{P}^{3}}^{1}(-4) \otimes \sigma_{*} \mathcal{O}_{\widetilde{\mathbb{P}}^{3}}(E) \cong \Omega_{\mathbb{P}^{3}}^{1}(-4), \\
R^{1} \sigma_{*}\left(\left(\sigma^{*} \Omega_{\mathbb{P}^{3}}^{1}\right) \otimes \mathcal{L}^{-1}\right) & \cong \Omega_{\mathbb{P}^{3}}^{1}(-4) \otimes R^{1} \sigma_{*} \mathcal{O}_{\widetilde{\mathbb{P}}^{3}}(E)=0 .
\end{aligned}
$$

Using again [7, Ex. III.8.4] and the projection formula we obtain

$$
\begin{aligned}
\sigma_{*}\left(\left(\Omega_{\mathbb{\mathbb { P }}^{3} / \mathbb{P}^{3}}^{1}\right)\right. & \left.\otimes \mathcal{L}^{-1}\right) \cong \bigwedge^{2} \mathcal{N}_{C \mid \mathbb{P}^{3}}^{\vee} \otimes \mathcal{O}_{\mathbb{P}^{3}}(-4) \otimes \sigma_{*} \mathcal{O}_{E}(-3)=0, \\
R^{1} \sigma_{*}\left(\left(\Omega_{\mathbb{P}^{3} / \mathbb{P}^{3}}^{1}\right) \otimes\right. & \left.\mathcal{L}^{-1}\right) \\
& \cong \bigwedge^{2} \mathcal{N}_{C \mid \mathbb{P}^{3}}^{\vee} \otimes \mathcal{O}_{\mathbb{P}^{3}}(-4) \otimes R^{1} \sigma_{*} \mathcal{O}_{E}(-3) \\
& \cong \bigwedge^{2} \mathcal{N}_{C \mid \mathbb{P}^{3}}^{\vee} \otimes \mathcal{O}_{\mathbb{P}^{3}}(-4) \otimes\left(\sigma_{*} \mathcal{O}_{E}(1)\right)^{\vee} \otimes\left(\bigwedge^{2} \mathcal{N}_{C \mid \mathbb{P}^{3}}^{\vee}\right)^{\vee} \\
& \cong \mathcal{O}_{\mathbb{P}^{3}}(-4) \otimes\left(\mathcal{O}_{\mathbb{P}^{3}}(d) \oplus \mathcal{O}_{\mathbb{P}^{3}}(e)\right) \otimes \mathcal{O}_{C} \\
& \cong\left(\mathcal{O}_{\mathbb{P}^{3}}(d-4) \oplus \mathcal{O}_{\mathbb{P}^{3}}(e-4)\right) \otimes \mathcal{O}_{C} .
\end{aligned}
$$

The exact sequence $(2)$ yields therefore

$$
\begin{aligned}
\sigma_{*}\left(\Omega_{\mathbb{\mathbb { P }}^{3}}^{1} \otimes \mathcal{L}^{-1}\right) & \cong \Omega_{\mathbb{P}^{3}}^{1}(-4), \\
R^{1} \sigma_{*}\left(\Omega_{\mathbb{\mathbb { P }}^{3}}^{1} \otimes \mathcal{L}^{-1}\right) & \cong\left(\mathcal{O}_{\mathbb{P}^{3}}(d-4) \oplus \mathcal{O}_{\mathbb{P}^{3}}(e-4)\right) \otimes \mathcal{O}_{C} .
\end{aligned}
$$

Calculating cohomologies of the right-hand sides of the above equations we can write the Leray spectral sequence:
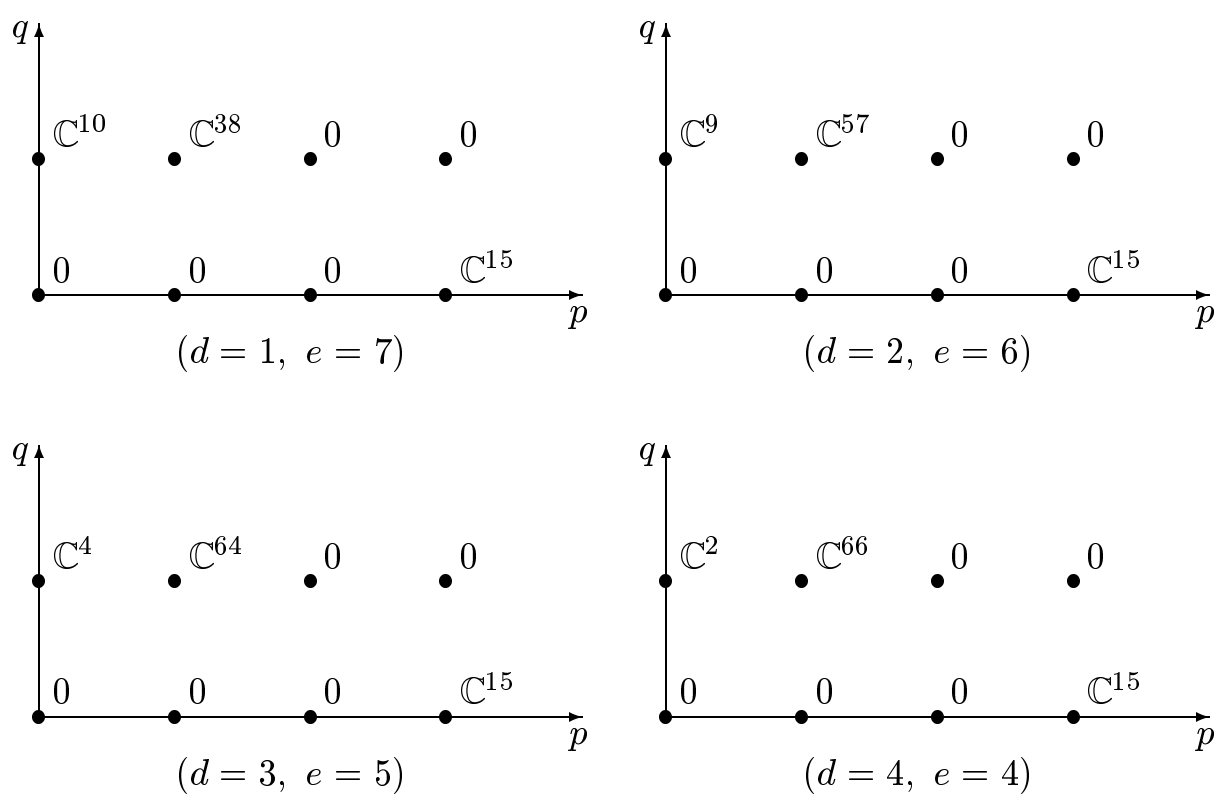

We can calculate $H^{0}$ and $H^{1}$ even if the sequence does not degenerate. This proves the lemma. 
We end this section with the following proposition:

Proposition 3.3.

$$
\begin{aligned}
& H^{0}\left(\pi^{*} \Omega_{\mathbb{\mathbb { P }}^{3}}^{1}\right)=0, \\
& H^{1}\left(\pi^{*} \Omega_{\mathbb{\mathbb { P }}^{3}}^{1}\right) \cong \begin{cases}\mathbb{C}^{12} & \text { if } d=1, e=7, \\
\mathbb{C}^{11} & \text { if } d=2, e=6, \\
\mathbb{C}^{6} & \text { if } d=3, e=5, \\
\mathbb{C}^{4} & \text { if } d=4, e=4 .\end{cases}
\end{aligned}
$$

Proof. Since $\pi$ is a double covering,

$$
\pi_{*} \mathcal{O}_{X} \cong \mathcal{O}_{\widetilde{\mathbb{P}}^{3}} \oplus \mathcal{L}^{-1} \text { and } H^{i}\left(\pi^{*} \Omega_{\widetilde{\mathbb{P}}^{3}}^{1}\right) \cong H^{i}\left(\pi_{*}\left(\pi^{*} \Omega_{\widetilde{\mathbb{P}}^{3}}^{1}\right)\right) .
$$

By the projection formula $\pi_{*} \pi^{*} \Omega_{\mathbb{\mathbb { P }}^{3}}^{1} \cong \Omega_{\widetilde{\mathbb{P}}^{3}}^{1} \otimes \pi_{*} \mathcal{O}_{X} \cong \Omega_{\mathbb{\mathbb { P }}^{3}}^{1} \oplus \Omega_{\mathbb{\mathbb { P }}^{3}}^{1} \otimes \mathcal{L}^{-1}$ and consequently

$$
H^{i}\left(\pi^{*} \Omega_{\mathbb{\mathbb { P }}^{3}}^{1}\right) \cong H^{i}\left(\Omega_{\mathbb{\mathbb { P }}^{3}}^{1}\right) \oplus H^{i}\left(\Omega_{\mathbb{\mathbb { P }}^{3}}^{1} \otimes \mathcal{L}^{-1}\right) .
$$

The proposition now follows from Lemmas 3.1 and 3.2.

4. Main result. Now we can formulate and prove our main result.

THEOREM 4.1.

$$
\begin{aligned}
& h^{1,1}(X)=2, \\
& h^{1,2}(X)= \begin{cases}122 & \text { if } d=1, e=7, \\
102 & \text { if } d=2, e=6, \\
90 & \text { if } d=3, e=5, \\
86 & \text { if } d=4, e=4 .\end{cases}
\end{aligned}
$$

The proof of this theorem is based on the following proposition:

Proposition 4.2 ([3]). The following sequence of $\mathcal{O}_{\tilde{X}}$-modules is exact:

$$
0 \rightarrow \pi^{*} \Omega_{\widetilde{\mathbb{P}} 3}^{1} \rightarrow \Omega_{X}^{1} \rightarrow \pi^{*}\left(\mathcal{O}_{\widetilde{B}} \otimes \mathcal{L}^{-1}\right) \rightarrow 0 .
$$

Proof of Theorem 4.1. By Lemma 2.1 the group $H^{1}\left(\mathcal{O}_{\widetilde{B}} \otimes \mathcal{L}^{-1}\right)$ vanishes. Since $X$ is a Calabi-Yau manifold, $H^{0}\left(\Omega^{1}(X)\right)=0$. Consequently, the long exact sequence derived from the short sequence (3) splits and its first part together with Lemma 2.1 and Proposition 3.3 gives $h^{1,1}=2$.

From the relation $e(X)=2\left(h^{1,1}-h^{1,2}\right)$ and the formula for $e(X)$ we compute $h^{1,2}(X)$.

Acknowledgements. Part of this work was done during the author's stay at Erlangen-Nürnberg University. The author would like to thank Prof. W. Barth for introducing him into this area of problems and numerous helpful discussions. 


\section{References}

[1] W. Barth, C. Peters and A. Van de Ven, Compact Complex Surfaces, Springer, Berlin, 1984.

[2] C. H. Clemens, Double solids, Adv. Math. 47 (1983), 107-230.

[3] S. Cynk, Hodge numbers of nodal double octics, Comm. Algebra 27 (1999), 4097-4102.

[4] -, Double octics with isolated singularities, Adv. Theor. Math. Phys. 3 (1999), $217-225$.

[5] S. Cynk and T. Szemberg, Double covers and Calabi-Yau varieties, in: Banach Center Publ. 44, Inst. Math., Polish Acad. Sci., 1998, 93-101.

[6] A. Dimca, Betti numbers of hypersurfaces and defects of linear systems, Duke Math. J. 60 (1990), 285-298.

[7] R. Hartshorne, Algebraic Geometry, Springer, Heidelberg, 1977.

Institute of Mathematics

Jagiellonian University

Reymonta 4

30-059 Kraków, Poland

E-mail: cynk@im.uj.edu.pl

Reçu par la Rédaction le 1.7.1999

Révisé le 20.10.1999 